\title{
REGRESI POISSON BIVARIAT DENGAN KOVARIAN MERUPAKAN FUNGSI DARI VARIABEL BEBAS*
}

\author{
Untung Kurniawan ${ }^{1}$ \\ 1Badan Pusat Statistik Kabupaten Klaten, Indonesia, untungk@bps.go.id \\ Indonesian Journal of Statistics and Its Applications (elSSN:2599-0802) \\ Vol 2 No 1 (2018), 23 - 34
}

Copyright ( 2018 Untung Kurniawan. This is an open-access article distributed under the Creative Commons Attribution License, which permits unrestricted use, distribution, and reproduction in any medium, provided the original work is properly cited.

\begin{abstract}
Poisson regression is a regression model which often used to analyze the count data. In this study, poisson regression has been used bivariate poisson regression where the regression is a method which is used to model a pair of correlated count data with multiple predictor variables. The model is used covariance which has a function of the independent variable. The purposes of this study is obtain parameter estimates, test statistics of bivariate poisson regression, and determine the factors that influence of infant mortality and maternal mortality. The data is used from the infant mortality and maternal mortality in Central Java 2015. Based on the result, the parameter estimation of poisson bivariate regression model using maximum likelihood (MLE) method. The results obtained from the parameter estimation are not close form so it needs to be done by Newton-Raphson iteration method. In testing the hypothesis using the Maximum Likelihood Ratio Test method (MLRT) by comparing the value between likelihood below $\mathrm{H}_{0}$ and likelihood below population. Partial of parameters model $\lambda_{1}$ (infant mortality) there are six independent variables that have significant influence, namely, delivery by health personnel $\left(X_{1}\right)$, pregnant women carry out the program K4 $\left(\mathrm{X}_{3}\right)$, pregnant women who get $\mathrm{Fe} 3$ tablet $\left(\mathrm{X}_{4}\right)$, handling obstetric complication $\left(X_{5}\right)$, exclusively breastfed infants $\left(X_{7}\right)$, and households living a clean and healthy life $\left(X_{8}\right)$. While for model $\lambda_{2}$ (maternal death) only variable handling of neonatal complication $\left(\mathrm{X}_{6}\right)$ which have no significant influence to response variable.
\end{abstract}

Keywords: bivariate poisson regression, infant mortality, maternal mortality, maximum likelihood estimation.

\section{Pendahuluan}

Seringkali suatu penelitian mengkaji hubungan antara variabel respon dan variabel bebas yang merupakan suatu teknik statistik yang disebut dengan analisis regresi.

\footnotetext{
* Received Jan 2018; Accepted Apr 2018; Published online on Apr 2018
} 
Sebagian besar analisis regresi menggunakan tipe data kontinu untuk variabel respon. Pada kenyataannya, banyak ditemukan kasus data dengan tipe diskrit pada variabel responnya (Long, 1997). Analisis yang tepat digunakan untuk menyelesaikan permasalahan tersebut adalah analisis regresi poisson. Suatu peristiwa akan mengikuti distribusi poisson jika peristiwa itu jarang sekali terjadi dalam suatu ruang sampel yang besar (Cameron dan Trivedi, 1998). Jumlah kematian bayi dan kematian ibu merupakan salah satu contoh data count yang mengikuti distribusi poisson. Untuk mengetahui faktor-faktor yang berpotensi dalam meningkatkan jumlah kasus kematian bayi dan ibu, dilakukan pemodelan dengan menggunakan analisis regresi poisson.

Model regresi data count bivariat digunakan ketika kejadian count yang secara bersama-sama saling bergantung (Gurmu dan Elder, 2007). Peristiwa count berpasangan yang menunjukkan korelasi harus ditaksir secara bersama, dan model regresi count bivariat dirancang untuk menangani kasus tersebut (Karlis dan Ntzoufras, 2005). Model regresi poisson bivariat banyak digunakan untuk data bivariat berkorelasi (Chou dan Steenhard, 2011). Kematian bayi dan kematian ibu merupakan dua hal yang saling berkaitan karena selama masa kandungan gizi yang diperoleh janin disalurkan dari tubuh ibu melalui plasenta sehingga kondisi ibu selama masa kehamilan akan berpengaruh pada janin dan bayi yang akan dilahirkannya kelak. Peran ibu juga sangat berpengaruh dalam merawat bayi mulai saat ia dilahirkan hingga berumur satu tahun.

Tujuan penelitian ini adalah mengkaji estimator parameter model regresi poison bivariat, mengkaji bentuk statistik uji model regresi poison bivariat, dan menentukan faktor-faktor yang berpengaruh terhadap jumlah kematian bayi dan jumlah kematian ibu di Propinsi Jawa Tengah Tahun 2015 melalui pendekatan regresi poison bivariat.

\section{Metodologi}

\subsection{Bahan dan Data}

Data yang digunakan dalam penelitian ini adalah data sekunder yang berasal dari data Profil Kesehatan Propinsi Jawa Tengah Tahun 2015 (Dinkes, 2015). Variabel respon pada penelitian ini adalah jumlah kematian bayi $\left(Y_{1}\right)$ dan jumlah kematian ibu $\left(\mathrm{Y}_{2}\right)$ tahun 2015 tiap kabupaten/kota di Provinsi Jawa Tengah. Variabel bebas pada penelitian ini adalah Persentase persalinan oleh tenaga kesehatan $\left(X_{1}\right)$, Persentase ibu bersalin mendapatkan pelayanan kesehatan nifas $\left(X_{2}\right)$, Persentase ibu hamil melaksanakan program K4 $\left(X_{3}\right)$, Persentase ibu hamil yang mendapatkan tablet $\mathrm{Fe} 3$ $\left(X_{4}\right)$, Persentase penanganan komplikasi kebidanan $\left(X_{5}\right)$, Persentase penanganan komplikasi neonatal $\left(X_{6}\right)$, Persentase bayi yang diberi ASI eksklusif $\left(X_{7}\right)$, Persentase rumah tangga berperilaku hidup bersih dan sehat $\left(\mathrm{X}_{8}\right)$.

\subsection{Metode Penelitian}

Langkah-langkah dalam analisis data untuk setiap tujuan penelitian adalah sebagai berikut :

1. Langkah-langkah untuk menentukan estimasi parameter pada model regresi poisson bivariat adalah sebagai berikut : 
1. Membentuk fungsi In likelihood dari model poisson bivariat dengan $\lambda_{0}$ merupakan fungsi dari variabel bebas yaitu

$$
\lambda_{0}=\exp \left(\beta_{00}+\beta_{01} x_{1}+\cdots+\beta_{0 k} x_{k}\right) .
$$

2. Melakukan transformasi ke dalam bentuk persamaan $\lambda_{j i}+\lambda_{0}=e^{\boldsymbol{x}_{i}{ }^{T} \boldsymbol{\beta}_{j}}$ terhadap fungsi In likelihood.

3. Menetapkan fungsi In likelihood $Q=\ln L\left(\boldsymbol{\beta}_{0}, \boldsymbol{\beta}_{1}, \boldsymbol{\beta}_{2}\right)$

4. Mencari turunan parsial pertama dari fungsi In likelihood

$$
\mathbf{g}^{T}(\boldsymbol{\theta})=\left(\left(\frac{\partial Q}{\partial \boldsymbol{\beta}_{0}}\right)^{T},\left(\frac{\partial Q}{\partial \boldsymbol{\beta}_{1}}\right)^{T},\left(\frac{\partial Q}{\partial \boldsymbol{\beta}_{2}}\right)^{T}\right)^{T}
$$

5. Mencari turunan parsial kedua dari fungsi In likelihood

$$
\mathbf{H}(\boldsymbol{\theta})=\left[\begin{array}{ccc}
\frac{\partial^{2} Q}{\partial \boldsymbol{\beta}_{0} \partial \boldsymbol{\beta}_{0}^{T}} & \frac{\partial^{2} Q}{\partial \boldsymbol{\beta}_{0} \partial \boldsymbol{\beta}_{1}} & \frac{\partial^{2} Q}{\partial \boldsymbol{\beta}_{0} \partial \boldsymbol{\beta}_{2}} \\
& \frac{\partial^{2} Q}{\partial \boldsymbol{\beta}_{1} \partial \boldsymbol{\beta}_{1}^{T}} & \frac{\partial^{2} Q}{\partial \boldsymbol{\beta}_{1} \partial \boldsymbol{\beta}_{2}} \\
\text { simetris } & & \frac{\partial^{2} Q}{\partial \boldsymbol{\beta}_{2} \partial \boldsymbol{\beta}_{2}^{T}}
\end{array}\right]
$$

6. Mendapatkan nilai penaksir parameter dengan iterasi NewtonRaphson.

2. Langkah-langkah untuk menentukan statistik uji pada model poisson bivariat adalah sebagai berikut :

Pengujian hipotesis secara serentak :

1. Membentuk hipotesis untuk menguji model regresi poisson bivariat:

$H_{0}: \beta_{j 1}=\beta_{j 2}=\ldots=\beta_{j 8}=0$

$H_{1}$ : paling sedikit ada satu $\beta_{j l} \neq 0 ; j=0,1,2 ; l=1,2, \ldots, 8$

2. Menentukan himpunan parameter-parameter di bawah $\mathrm{H}_{0}(\omega)$

3. Membuat fungsi likelihood di bawah $H_{0} L(\omega)$

4. Menentukan himpunan parameter-parameter di bawah populasi $(\Omega)$

5. Membuat fungsi likelihood di bawah populasi $L(\Omega)$

6. Menentukan penaksir parameter dengan metode MLE dan diperoleh $(\hat{\omega}) \operatorname{dan}(\hat{\Omega})$

7. Menentukan statistik uji dengan menggunakan metode Maximum Likelihood Ratio Test (MLRT)

8. Menentukan daerah penolakan $\mathrm{H}_{0}$

Pengujian hipotesis secara parsial adalah sebagai berikut:

1. Hipotesis untuk menguji signifikansi parameter $\beta$

$$
\begin{aligned}
& H_{0}: \beta_{j l}=0 \\
& H_{1}: \beta_{j l} \neq 0 ; j=0,1,2 ; l=1,2, \ldots, 8
\end{aligned}
$$

2. Menentukan statistik uji.

$$
z=\frac{\hat{\beta}_{j l}}{\operatorname{se}\left(\hat{\beta}_{j l}\right)}
$$


3. Menentukan daerah penolakan $\mathrm{H}_{0}$

Daerah penolakan $\mathrm{H}_{0}$ adalah $\mid z$ hitung $\mid$ lebih besar dari $z_{\alpha / 2}$. Nilai se $\left(\hat{\beta}_{j l}\right)$ diperoleh dari diagonal ke $(j+1)$ dari $\operatorname{var}(\hat{\boldsymbol{\theta}})$.

3. Langkah-langkah untuk menentukan faktor-faktor yang berpengaruh terhadap kematian bayi dan kematian ibu di Provinsi Jawa Tengah dengan pendekatan model regresi poison bivariat adalah sebagai berikut :

1. Melakukan estimasi parameter model regresi poisson bivariat dengan menggunakan Maximum Likelihood Estimation (MLE) di mana nilai $\lambda_{0}$ merupakan fungsi dari variabel bebas.

2. Melakukan pengujian hipotesis untuk regresi poison bivariat.

3. Melakukan interpretasi model yang didapatkan.

\section{Hasil dan Pembahasan}

\subsection{Estimasi Parameter Regresi Poison Bivariat dengan Kovarian merupakan Fungsi dari Variabel Bebas.}

Regresi Poisson merupakan model regresi yang sering digunakan untuk menganalisis suatu data count. Regresi poisson mengacu pada penggunaan distribusi poisson. Suatu metode yang digunakan untuk memodelkan sepasang count data yang memiliki korelasi (Karlis dan Ntzoufras, 2005) dengan beberapa variabel prediktor adalah regresi poisson bivariat. Model tersebut seperti pada persamaan berikut:

$$
\begin{aligned}
& \left(Y_{1 i}, Y_{2 i}\right) \sim P B\left(\lambda_{1 i}, \lambda_{2 i}, \lambda_{0}\right) \\
& \lambda_{j i}+\lambda_{0}=e^{x_{i}^{T} \beta_{j}} ; j=1,2 \\
& \mathbf{x}_{i}=\left[\begin{array}{lllll}
1 & x_{1 i} & x_{2 i} & \ldots & x_{k i}
\end{array}\right]^{T} \\
& \boldsymbol{\beta}_{j}=\left[\begin{array}{lllll}
\beta_{j 0} & \beta_{j 1} & \beta_{j 2} & \ldots & \beta_{j k}
\end{array}\right]^{T}
\end{aligned}
$$

dimana $i=1,2, \cdots, n$, menunjukkan nomor observasi, observasi digunakan untuk model $\lambda_{i}$ dan $\beta_{j}$ menunjukkan vektor korespondensi dari koefisien regresi. Terdapat tiga buah model dengan nilai $\lambda_{0}$ yang berbeda, yaitu

a) Model dengan nilai $\lambda_{0}$ adalah suatu konstanta.

b) Model dengan nilai $\lambda_{0}$ merupakan fungsi dari variabel bebas sehingga persamaannya sebagai berikut :

$\lambda_{0}=\exp \left(\beta_{00}+\beta_{01} x_{1}+\cdots+\beta_{0 k} x_{k}\right)$.

c) Model dengan nilai $\lambda_{0}$ adalah nol dimana tidak ada kovarian dari kedua buah variabel tersebut.

Pada penelitian ini digunakan model $\lambda_{0}$ merupakan fungsi dari variabel bebas sehingga persamaannya sebagai berikut :

$$
\lambda_{0}=\exp \left(\beta_{00}+\beta_{01} x_{1}+\cdots+\beta_{0 k} x_{k}\right) .
$$

Metode estimasi yang digunakan dalam regresi poisson bivariat adalah Maximum Likelihood Estimation (MLE) dengan fungsi likelihoodnya sebagai berikut : 


$$
\begin{aligned}
& L\left(\lambda_{1}, \lambda_{2}, \lambda_{0}\right)=\prod_{i=1}^{n}\left(e^{-\left(\lambda_{0}+\lambda_{1 i}+\lambda_{2 i}\right)} \sum_{k=0}^{s} \frac{\lambda_{1 i}^{y_{1 i}-k} \lambda_{2 i}^{y_{2 i}-k} \lambda_{0}^{k}}{\left(y_{1 i}-k\right) !\left(y_{2 i}-k\right) !(k) !}\right) \\
& s=\min \left(y_{1}, y_{2}\right)
\end{aligned}
$$

Selanjutnya ditransformasi dengan $\lambda_{j i}+\lambda_{0}=e^{\boldsymbol{x}_{i}{ }^{T} \boldsymbol{\beta}_{j}}$ sehingga diperoleh fungsi In likelihood yang baru yaitu :

$$
\begin{array}{r}
L\left(\boldsymbol{\beta}_{\mathbf{0}}, \boldsymbol{\beta}_{\mathbf{1}}, \boldsymbol{\beta}_{\mathbf{2}}\right)=\prod_{i=1}^{n}\left(\exp \left(-e^{\mathbf{x}_{\mathbf{i}}^{T} \boldsymbol{\beta}_{\mathbf{0}}}\right)-\exp \left(e^{\mathbf{x}_{\mathbf{i}}^{T} \boldsymbol{\beta}_{\mathbf{1}}}-e^{\mathbf{x}_{\mathbf{i}}^{T} \boldsymbol{\beta}_{\mathbf{0}}}\right)-\exp \left(e^{\mathbf{x}_{\mathbf{i}}^{T} \boldsymbol{\beta}_{2}}-e^{\mathbf{x}^{T} \boldsymbol{\beta}_{\mathbf{0}}}\right)\right) \cdot W_{i} \\
\text { dengan } W_{i}=\sum_{k=0}^{\min \left(y_{1 i}, y_{2 i}\right)} \frac{\left(e^{\mathbf{x}_{\mathbf{i}}^{T} \boldsymbol{\beta}_{\mathbf{1}}}-e^{\mathbf{x}_{\mathbf{i}}^{T} \boldsymbol{\beta}_{\mathbf{0}}}\right)^{y_{1 i}-k}\left(e^{\mathbf{x}_{\mathbf{i}}^{T} \boldsymbol{\beta}_{2}}-e^{\mathbf{x}_{\mathbf{i}}^{T} \boldsymbol{\beta}_{\mathbf{0}}}\right)^{y_{2 i}-k}}{\left(y_{1 i}-k\right) !\left(y_{2 i}-k\right) !} \frac{\left(e^{\mathbf{x}_{\mathbf{i}}^{T} \boldsymbol{\beta}_{\mathbf{0}}}\right)^{k}}{k !}
\end{array}
$$

sehingga fungsi In likelihood diperoleh sebagai berikut :

$$
Q=\ln L\left(\boldsymbol{\beta}_{\mathbf{0}}, \boldsymbol{\beta}_{\mathbf{1}}, \boldsymbol{\beta}_{\mathbf{2}}\right)=-\sum_{i=1}^{n}\left(e^{\mathbf{x}_{\mathbf{i}}^{T} \mathbf{\beta}_{\mathbf{0}}}\right)-\sum_{i=1}^{n}\left(e^{\mathbf{x}_{\mathbf{i}}^{T} \mathbf{\beta}_{1}}-e^{\mathbf{x}_{\mathbf{i}}^{T} \mathbf{\beta}_{\mathbf{0}}}\right)-\sum_{i=1}^{n}\left(e^{\mathbf{x}_{\mathbf{i}}^{T} \boldsymbol{\beta}_{2}}-e^{\mathbf{x}_{\mathbf{i}}^{T} \mathbf{\beta}_{\mathbf{0}}}\right)+\sum_{i=1}^{n} \ln W_{i}
$$

misalkan $W_{i}=\sum_{k=0}^{\min \left(y_{1 i}, y_{2 i}\right)} W_{1 i} \cdot W_{2 i}$

$$
\begin{aligned}
W_{1 i} & =\frac{\left(e^{\mathbf{x}_{\mathbf{i}}^{T} \boldsymbol{\beta}_{\mathbf{1}}}-e^{\mathbf{x}_{\mathbf{i}}^{T} \boldsymbol{\beta}_{\mathbf{0}}}\right)^{y_{1 i}-k}}{\left(y_{1 i}-k\right) !} \\
W_{2 i} & =\frac{\left(e^{\mathbf{x}_{\mathbf{i}}^{T} \boldsymbol{\beta}_{2}}-e^{\mathbf{x}_{\mathbf{i}}^{T} \boldsymbol{\beta}_{\mathbf{0}}}\right)^{y_{2 i}-k}\left(e^{\mathbf{x}_{\mathbf{i}}^{T} \boldsymbol{\beta}_{\mathbf{0}}}\right)^{k}}{\left(y_{2 i}-k\right) ! k !}
\end{aligned}
$$

$Q$ diturunkan terhadap $\boldsymbol{\beta}_{0}, \boldsymbol{\beta}_{1}, \boldsymbol{\beta}_{2}$

$$
\begin{aligned}
& \frac{\partial Q}{\partial \boldsymbol{\beta}_{0}}=\sum_{i=1}^{n} \exp \left(\mathbf{x}_{\mathbf{i}}{ }^{T} \boldsymbol{\beta}_{\mathbf{0}}\right) \mathbf{x}_{\mathbf{i}}+\sum_{i=1}^{n} \frac{1}{W_{i}} \frac{\partial W_{i}}{\partial \boldsymbol{\beta}_{\mathbf{0}}} \\
& \frac{\partial Q}{\partial \boldsymbol{\beta}_{\mathbf{1}}}=-\sum_{i=1}^{n} \exp \left(\mathbf{x}_{\mathbf{i}}{ }^{T} \boldsymbol{\beta}_{\mathbf{1}}\right) \mathbf{x}_{\mathbf{i}}+\sum_{i=1}^{n} \frac{1}{W_{i}} \frac{\partial W_{i}}{\partial \boldsymbol{\beta}_{\mathbf{1}}} \\
& \frac{\partial Q}{\partial \boldsymbol{\beta}_{\mathbf{2}}}=-\sum_{i=1}^{n} \exp \left(\mathbf{x}_{\mathbf{i}}{ }^{T} \boldsymbol{\beta}_{\mathbf{2}}\right) \mathbf{x}_{\mathbf{i}}+\sum_{i=1}^{n} \frac{1}{W_{i}} \frac{\partial W_{i}}{\partial \boldsymbol{\beta}_{\mathbf{2}}}
\end{aligned}
$$

$W_{1 i}$ diturunkan terhadap $\boldsymbol{\beta}_{0}$

$$
\begin{aligned}
& \frac{\partial W_{1 i}}{\partial \boldsymbol{\beta}_{0}}=\frac{\left(y_{1 i}-k\right)\left(e^{\mathbf{x}_{\mathbf{i}}^{T} \boldsymbol{\beta}_{1}}-e^{\mathbf{x}_{\mathbf{i}}^{T} \boldsymbol{\beta}_{0}}\right)^{y_{1 i}-k-1}\left(-e^{\mathbf{x}_{\mathbf{i}}^{T} \boldsymbol{\beta}_{0}}\right) \mathbf{x}_{\mathbf{i}}}{\left(y_{1 i}-k\right) !} \\
& \frac{\partial W_{1 i}}{\partial \boldsymbol{\beta}_{0}}=\frac{\left(y_{1 i}-k\right)\left(e^{\mathbf{x}_{\mathbf{i}}^{T} \boldsymbol{\beta}_{1}}-e^{\mathbf{x}_{\mathbf{i}}{ }^{T} \boldsymbol{\beta}_{0}}\right)^{y_{1 i}-k-1}\left(-e^{\mathbf{x}_{\mathbf{i}}{ }^{T} \boldsymbol{\beta}_{0}}\right) \mathbf{x}_{\mathbf{i}}}{\left(y_{1 i}-k\right)\left(y_{1 i}-k-1\right) !} \\
& \frac{\partial W_{1 i}}{\partial \boldsymbol{\beta}_{0}}=\frac{\left(e^{\mathbf{x}_{\mathbf{i}}{ }^{T} \boldsymbol{\beta}_{1}}-e^{\mathbf{x}_{\mathbf{i}} \boldsymbol{\beta}_{\mathbf{0}}}\right)^{y_{1 i}-k-1}\left(-e^{\mathbf{x}_{\mathbf{i}} \boldsymbol{\beta}_{\mathbf{0}}}\right) \mathbf{x}_{\mathbf{i}}}{\left(y_{1 i}-k-1\right) !}
\end{aligned}
$$

$W_{2 i}$ diturunkan terhadap $\boldsymbol{\beta}_{0}$

$$
\begin{aligned}
W_{2 i} & =\frac{\left(e^{\mathbf{x}_{\mathbf{i}}^{T} \boldsymbol{\beta}_{2}}-e^{\mathbf{x}_{\mathbf{i}}^{T} \boldsymbol{\beta}_{\mathbf{0}}}\right)^{y_{2 i}-k}\left(e^{\mathbf{x}_{\mathbf{i}}^{T} \mathbf{\beta}_{\mathbf{0}}}\right)^{k}}{\left(y_{2 i}-k\right) ! k !} \\
\frac{\partial W_{2 i}}{\partial \boldsymbol{\beta}_{0}} & =u^{\prime} v+v^{\prime} u
\end{aligned}
$$


dengan,

$$
\begin{aligned}
& u=\frac{\left(e^{\mathbf{x}_{\mathbf{i}}^{T} \boldsymbol{\beta}_{2}}-e^{\mathbf{x}_{\mathbf{i}}^{T} \mathbf{\beta}_{\mathbf{0}}}\right)^{y_{2 i}-k}}{\left(y_{2 i}-k\right) !} \\
& v=\frac{\left(e^{\mathbf{x}_{\mathbf{i}}^{T} \mathbf{\beta}_{\mathbf{0}}}\right)^{k}}{k !} \\
& u^{\prime}=\frac{\left(y_{2 i}-k\right)\left(e^{\mathbf{x}_{\mathbf{i}}^{T} \mathbf{\beta}_{2}}-e^{\mathbf{x}_{\mathbf{i}}^{T} \mathbf{\beta}_{\mathbf{0}}}\right)^{y_{2 i}-k-1}\left(-e^{\mathbf{x}_{\mathbf{i}}^{T} \mathbf{\beta}_{\mathbf{0}}}\right) \mathbf{x}_{\mathbf{i}}}{\left(y_{2 i}-k\right) !} \\
& v^{\prime}=\frac{k\left(e^{\mathbf{x}_{\mathbf{i}}{ }^{T} \mathbf{B}_{0}}\right)^{k-1} \mathbf{x}_{\mathbf{i}}}{k !} \\
& \frac{\partial W_{2 i}}{\partial \boldsymbol{\beta}_{0}}=\frac{\left(y_{2 i}-k\right)\left(e^{\mathbf{x}_{\mathbf{i}}^{T} \boldsymbol{\beta}_{2}}-e^{\mathbf{x}_{\mathbf{i}}^{T} \boldsymbol{\beta}_{\mathbf{0}}}\right)^{y_{2 i}-k-1}\left(-e^{\mathbf{x}_{\mathbf{i}}^{T} \boldsymbol{\beta}_{\mathbf{0}}}\right) \mathbf{x}_{\mathbf{i}}}{\left(y_{2 i}-k\right) !} \cdot \frac{\left(e^{\mathbf{x}_{\mathbf{i}}{ }^{T} \boldsymbol{\beta}_{\mathbf{0}}}\right)^{k}}{k !}+\frac{k\left(e^{\mathbf{x}_{\mathbf{i}}^{T} \boldsymbol{\beta}_{\mathbf{0}}}\right)^{k-1} \mathbf{x}_{\mathbf{i}}}{k !} \cdot \frac{\left(e^{\mathbf{x}_{\mathbf{i}}^{T} \boldsymbol{\beta}_{2}}-e^{\mathbf{x}_{\mathbf{i}}^{T} \mathbf{\beta}_{\mathbf{0}}}\right)^{y_{2 i}-k}}{\left(y_{2 i}-k\right) !} \\
& =\frac{\left(e^{\mathbf{x}_{\mathbf{i}}^{T} \boldsymbol{\beta}_{2}}-e^{\mathbf{x}_{\mathbf{i}}^{T} \boldsymbol{\beta}_{0}}\right)^{y_{2 i}-k}\left(e^{\mathbf{x}_{\mathbf{i}}^{T} \mathbf{\beta}_{0}}\right)^{k}}{\left(y_{2 i}-k\right) ! k !} \cdot\left[\frac{\left(y_{2 i}-k\right)\left(-e^{\mathbf{x}_{\mathbf{i}}^{T} \mathbf{\beta}_{\mathbf{0}}}\right) \mathbf{x}_{\mathbf{i}}}{\left(e^{\mathbf{x}_{\mathbf{i}}^{T} \boldsymbol{\beta}_{2}}-e^{\mathbf{x}_{\mathbf{i}}^{T} \boldsymbol{\beta}_{\mathbf{0}}}\right)}+\frac{k \mathbf{x}_{\mathbf{i}}}{e^{\mathbf{x}_{\mathbf{i}} \mathbf{\beta}_{\mathbf{0}}}}\right] \\
& \frac{\partial W_{i}}{\partial \boldsymbol{\beta}_{\mathbf{0}}}=\sum_{k=0}^{\min \left(y_{y_{i}}, y_{2 i}\right)}\left(\frac{\partial W_{1 i}}{\partial \boldsymbol{\beta}_{\mathbf{0}}} W_{2 i}+\frac{\partial W_{2 i}}{\partial \boldsymbol{\beta}_{\mathbf{0}}} W_{1 i}\right) \\
& =\sum_{k=0}^{\min \left(y_{1 i}, y_{2 i}\right)} \frac{\left(y_{1 i}-k\right)\left(e^{\mathbf{x}_{\mathbf{i}} \boldsymbol{\beta}_{1}}-e^{\mathbf{x}_{\mathbf{i}}^{T} \boldsymbol{\beta}_{0}}\right)^{y_{1 i}-k-1}\left(-e^{\mathbf{x}_{\mathbf{i}}^{T} \boldsymbol{\beta}_{0}}\right) \mathbf{x}_{\mathbf{i}}}{\left(y_{1 i}-k\right) !} \cdot \frac{\left(e^{\mathbf{x}_{\mathbf{i}}{ }^{T} \boldsymbol{\beta}_{2}}-e^{\mathbf{x}_{i}^{T} \mathbf{\beta}_{0}}\right)^{y_{2 i}-k}\left(e^{\mathbf{x}_{\mathbf{i}} \boldsymbol{\beta}_{0}}\right)^{k}}{\left(y_{2 i}-k\right) ! k !}
\end{aligned}
$$

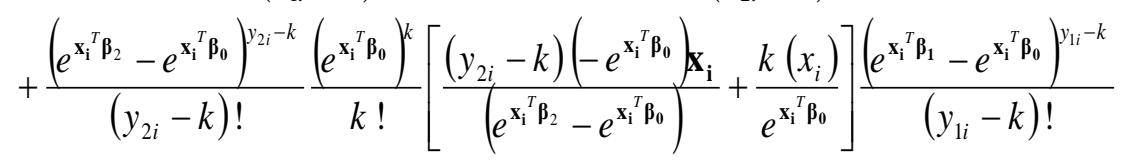

$$
\begin{aligned}
& =\sum_{k=0}^{\min \left(y_{1 i}, y_{2 i}\right)} \frac{\left(e^{\mathbf{x}_{i}^{T} \boldsymbol{\beta}_{1}}-e^{\mathbf{x}_{i}^{T} \mathbf{\beta}_{0}}\right)^{y_{1 i}-k}}{\left(y_{1 i}-k\right) !} \cdot \frac{\left(e^{\mathbf{x}_{\mathbf{i}}^{T} \boldsymbol{\beta}_{2}}-e^{\mathbf{x}_{i}^{T} \mathbf{\beta}_{0}}\right)^{y_{2 i}-k}\left(e^{\mathbf{x}_{\mathbf{i}}^{T} \mathbf{\beta}_{0}}\right)^{k}}{\left(y_{2 i}-k\right) ! k !} \\
& {\left[\frac{\left(y_{1 i}-k\right)\left(-e^{\mathbf{x}_{\mathbf{i}}^{T} \boldsymbol{\beta}_{0}}\right) \mathbf{x}_{\mathbf{i}}}{e^{\mathbf{x}_{\mathbf{i}}^{T} \boldsymbol{\beta}_{1}}-e^{\mathbf{x}_{\mathbf{i}}^{T} \boldsymbol{\beta}_{0}}}+\frac{\left(y_{2 i}-k\right)\left(-e^{\mathbf{x}_{\mathbf{i}}^{T} \boldsymbol{\beta}_{0}}\right) \mathbf{x}_{\mathbf{i}}}{\left(e^{\mathbf{x}_{\mathbf{i}}^{T} \boldsymbol{\beta}_{2}}-e^{\mathbf{x}_{\mathbf{i}}^{T} \boldsymbol{\beta}_{0}}\right)}+\frac{k \mathbf{x}_{\mathbf{i}}}{e^{\mathbf{x}_{\mathbf{i}}^{T} \mathbf{\beta}_{0}}}\right]}
\end{aligned}
$$

$W_{1 i}$ diturunkan terhadap $\boldsymbol{\beta}_{1}$

$$
\begin{aligned}
& \frac{\partial W_{1 i}}{\partial \boldsymbol{\beta}_{\mathbf{1}}}=\frac{\left(y_{1 i}-k\right)\left(e^{\mathbf{x}_{\mathbf{i}}^{T} \boldsymbol{\beta}_{\mathbf{1}}}-e^{\mathbf{x}_{\mathbf{i}}^{T} \boldsymbol{\beta}_{\mathbf{0}}}\right)^{y_{1 i}-k-1}\left(e^{\mathbf{x}_{\mathbf{i}}^{T} \boldsymbol{\beta}_{\mathbf{1}}}\right) \mathbf{x}_{\mathbf{i}}}{\left(y_{1 i}-k\right) !} \\
& \frac{\partial W_{1 i}}{\partial \boldsymbol{\beta}_{\mathbf{1}}}=\frac{\left(y_{1 i}-k\right)\left(e^{\mathbf{x}_{\mathbf{i}}^{T} \boldsymbol{\beta}_{\mathbf{1}}}-e^{\mathbf{x}_{\mathbf{i}}^{T} \boldsymbol{\beta}_{\mathbf{0}}}\right)^{y_{1 i}-k-1}\left(e^{\mathbf{x}_{\mathbf{i}}^{T} \boldsymbol{\beta}_{\mathbf{1}}}\right) \mathbf{x}_{\mathbf{i}}}{\left(y_{1 i}-k\right)\left(y_{1 i}-k-1\right) !} \\
& \frac{\partial W_{1 i}}{\partial \boldsymbol{\beta}_{\mathbf{1}}}=\frac{\left(e^{\mathbf{x}_{\mathbf{i}}{ }^{T} \boldsymbol{\beta}_{\mathbf{1}}}-e^{\mathbf{x}_{\mathbf{i}}^{T} \boldsymbol{\beta}_{\mathbf{0}}}\right)^{y_{1 i}-k-1}\left(e^{\mathbf{x}_{\mathbf{i}}{ }^{T} \boldsymbol{\beta}_{\mathbf{1}}}\right) \mathbf{x}_{\mathbf{i}}}{\left(y_{1 i}-k-1\right) !}
\end{aligned}
$$

$W_{2 i}$ diturunkan terhadap $\boldsymbol{\beta}_{1}$

$$
\frac{\partial W_{2 i}}{\partial \boldsymbol{\beta}_{1}}=0
$$




$$
\begin{aligned}
& \frac{\partial W_{i}}{\partial \boldsymbol{\beta}_{1}}=\sum_{k=0}^{\min \left(y_{1 i}, y_{2 i}\right)}\left(\frac{\partial W_{1 i}}{\partial \boldsymbol{\beta}_{1}} W_{2 i}+\frac{\partial W_{2 i}}{\partial \boldsymbol{\beta}_{1}} W_{1 i}\right) \\
& \frac{\partial W_{i}}{\partial \boldsymbol{\beta}_{1}}=\sum_{k=0}^{\min \left(y_{1 i}, y_{2 i}\right)} \frac{\left(e^{\mathbf{x}_{\mathbf{i}}^{T} \mathbf{\beta}_{1}}-e^{\mathbf{x}_{\mathbf{i}}^{T} \boldsymbol{\beta}_{\mathbf{0}}}\right)^{y_{1 i}-k-1}\left(e^{\mathbf{x}_{\mathbf{i}}^{T} \boldsymbol{\beta}_{1}}\right) \mathbf{x}_{\mathbf{i}}}{\left(y_{1 i}-k-1\right) !} \cdot \frac{\left(e^{\mathbf{x}_{\mathbf{i}} \mathbf{\beta}_{2}}-e^{\mathbf{x}_{\mathbf{i}}{ }^{T} \boldsymbol{\beta}_{\mathbf{0}}}\right)^{y_{2 i}-k}\left(e^{\mathbf{x}_{\mathbf{i}}{ }^{T} \boldsymbol{\beta}_{\mathbf{0}}}\right)^{k}}{\left(y_{2 i}-k\right) ! k !}
\end{aligned}
$$

$W_{1 i}$ diturunkan terhadap $\boldsymbol{\beta}_{2}$

$$
\frac{\partial W_{1 i}}{\partial \boldsymbol{\beta}_{2}}=0
$$

$W_{2 i}$ diturunkan terhadap $\boldsymbol{\beta}_{2}$

$$
\begin{aligned}
\frac{\partial W_{2 i}}{\partial \boldsymbol{\beta}_{2}} & =\frac{\left(y_{2 i}-k\right)\left(e^{\mathbf{x}_{\mathbf{i}}^{T} \boldsymbol{\beta}_{2}}-e^{\mathbf{x}_{\mathbf{i}}^{T} \boldsymbol{\beta}_{\mathbf{0}}}\right)^{y_{1 i}-k-1}\left(e^{\mathbf{x}_{\mathbf{i}}^{T} \boldsymbol{\beta}_{2}}\right) \mathbf{x}_{\mathbf{i}}}{\left(y_{2 i}-k\right) !} \cdot \frac{\left(e^{\mathbf{x}_{\mathbf{i}}^{T} \boldsymbol{\beta}_{\mathbf{0}}}\right)^{k}}{k !} \\
\frac{\partial W_{i}}{\partial \boldsymbol{\beta}_{2}} & =\sum_{k=0}^{\min \left(y_{1 i}, y_{2 i}\right)}\left(\frac{\partial W_{1 i}}{\partial \boldsymbol{\beta}_{2}} W_{2 i}+\frac{\partial W_{2 i}}{\partial \boldsymbol{\beta}_{2}} W_{1 i}\right) \\
& =\sum_{k=0}^{\min \left(y_{1 i}, y_{2 i}\right.} \frac{\left(y_{2 i}-k\right)\left(e^{\mathbf{x}_{\mathbf{i}}^{T} \boldsymbol{\beta}_{2}}-e^{\mathbf{x}_{\mathbf{i}}^{T} \boldsymbol{\beta}_{\mathbf{0}}}\right)^{y_{1 i}-k-1}\left(e^{\mathbf{x}_{\mathbf{i}}^{T} \boldsymbol{\beta}_{2}}\right) \mathbf{x}_{\mathbf{i}}}{\left(y_{1 i}-k\right) !} \cdot \frac{\left(e^{\mathbf{x}_{\mathbf{i}}^{T} \boldsymbol{\beta}_{\mathbf{0}}}\right)^{k}\left(e^{\mathbf{x}_{\mathbf{i}}^{T} \boldsymbol{\beta}_{1}}-e^{\mathbf{x}_{\mathbf{i}}^{T} \boldsymbol{\beta}_{\mathbf{0}}}\right)^{y_{1 i}-k}}{\left(y_{2 i}-k\right) ! k !}
\end{aligned}
$$

Turunan kedua sebagai berikut :

$$
\begin{aligned}
\frac{\partial^{2} Q}{\partial \boldsymbol{\beta}_{\mathbf{0}} \partial \boldsymbol{\beta}_{0}^{T}}=\sum_{i=1}^{n} \exp \left(\mathbf{x}_{\mathbf{i}}{ }^{T} \boldsymbol{\beta}_{\mathbf{0}}\right) \mathbf{x}_{\mathbf{i}} \mathbf{x}_{\mathbf{i}}^{\mathbf{T}}+\sum_{i=1}^{n}\left[\left(\frac{1}{W_{i}} \frac{\partial^{2} W_{i}}{\partial \boldsymbol{\beta}_{\mathbf{0}} \partial \boldsymbol{\beta}_{0}^{T}}\right)-\left(\frac{1}{W_{i}} \frac{\partial W_{i}}{\partial \boldsymbol{\beta}_{\mathbf{0}}} \frac{\partial W_{i}}{\partial \boldsymbol{\beta}_{0}^{T}}\right)\right] \\
\frac{\partial^{2} Q}{\partial \boldsymbol{\beta}_{\mathbf{1}} \partial \boldsymbol{\beta}_{1}^{T}}=-\sum_{i=1}^{n} \exp \left(\mathbf{x}_{i}{ }^{T} \boldsymbol{\beta}_{1}\right) \mathbf{x}_{\mathbf{i}} \mathbf{x}_{\mathbf{i}}^{\mathbf{T}}+\sum_{i=1}^{n}\left[\left(\frac{1}{W_{i}} \frac{\partial^{2} W_{i}}{\partial \boldsymbol{\beta}_{\mathbf{1}} \partial \boldsymbol{\beta}_{1}^{T}}\right)-\left(\frac{1}{W_{i}{ }^{2}} \frac{\partial W_{i}}{\partial \boldsymbol{\beta}_{\mathbf{1}}} \frac{\partial W_{i}}{\partial \boldsymbol{\beta}_{1}^{T}}\right)\right] \\
\frac{\partial^{2} Q}{\partial \boldsymbol{\beta}_{\mathbf{2}} \partial \boldsymbol{\beta}_{2}^{T}}=-\sum_{i=1}^{n} \exp \left(\mathbf{x}_{i}{ }^{T} \boldsymbol{\beta}_{2}\right) \mathbf{x}_{\mathbf{i}} \mathbf{x}_{\mathbf{i}}^{\mathbf{T}}+\sum_{i=1}^{n}\left[\left(\frac{1}{W_{i}} \frac{\partial^{2} W_{i}}{\partial \boldsymbol{\beta}_{\mathbf{2}} \partial \boldsymbol{\beta}_{2}^{T}}\right)-\left(\frac{1}{W_{i}{ }^{2}} \frac{\partial W_{i}}{\partial \boldsymbol{\beta}_{\mathbf{2}}} \frac{\partial W_{i}}{\partial \boldsymbol{\beta}_{2}^{T}}\right)\right] \\
\frac{\partial^{2} Q}{\partial \boldsymbol{\beta}_{\mathbf{0}} \partial \boldsymbol{\beta}_{\mathbf{1}}}=\sum_{i=1}^{n}\left[\left(\frac{1}{W_{i}} \frac{\partial^{2} W_{i}}{\partial \boldsymbol{\beta}_{\mathbf{0}} \partial \boldsymbol{\beta}_{\mathbf{1}}}\right)-\left(\frac{1}{W_{i}^{2}} \frac{\partial W_{i}}{\partial \boldsymbol{\beta}_{\mathbf{0}}} \frac{\partial W_{i}}{\partial \boldsymbol{\beta}_{1}}\right)\right] \\
\frac{\partial^{2} Q}{\partial \boldsymbol{\beta}_{\mathbf{0}} \partial \boldsymbol{\beta}_{\mathbf{2}}}=\sum_{i=1}^{n}\left[\left(\frac{1}{W_{i}} \frac{\partial^{2} W_{i}}{\partial \boldsymbol{\beta}_{\mathbf{0}} \partial \boldsymbol{\beta}_{\mathbf{2}}}\right)-\left(\frac{1}{W_{i}^{2}} \frac{\partial W_{i}}{\partial \boldsymbol{\beta}_{\mathbf{0}}} \frac{\partial W_{i}}{\partial \boldsymbol{\beta}_{\mathbf{2}}}\right)\right] \\
\frac{\partial^{2} Q}{\partial \boldsymbol{\beta}_{\mathbf{1}} \partial \boldsymbol{\beta}_{\mathbf{2}}}=-\sum_{i=1}^{n}\left[\left(\frac{1}{W_{i}} \frac{\partial^{2} W_{i}}{\partial \boldsymbol{\beta}_{\mathbf{1}} \partial \boldsymbol{\beta}_{\mathbf{2}}}\right)-\left(\frac{1}{W_{i}^{2}} \frac{\partial W_{i}}{\partial \boldsymbol{\beta}_{\mathbf{1}}} \frac{\partial W_{i}}{\partial \boldsymbol{\beta}_{\mathbf{2}}}\right)\right]
\end{aligned}
$$

Karena hasil persamaan di atas tidak memberikan suatu persamaan yang eksplisit maka digunakan suatu metode yaitu metode Newton-Rapshon dengan langkah-langkah sebagai berikut :

1. Menentukan nilai taksiran awal parameter $\hat{\boldsymbol{\theta}}_{(\mathbf{0})}$ dengan $\boldsymbol{\theta}=\left(\lambda_{0} \boldsymbol{\beta}_{1}^{\mathbf{T}} \boldsymbol{\beta}_{2}^{\mathbf{T}}\right)^{T}$ di mana $\ln L(\boldsymbol{\theta})=Q$. Niai taksiran awal parameter $\hat{\lambda}_{0(0)}$ dapat digunakan $\operatorname{cov}\left[\mathrm{Y}_{1}, \mathrm{Y}_{2}\right]=\lambda_{0}$. Nilai taksiran awal $\hat{\boldsymbol{\beta}}_{\mathbf{j}(\mathbf{0})}$ diperoleh dengan metode Ordinary Least square (OLS), yaitu

$\hat{\boldsymbol{\beta}}_{\mathbf{j}(\mathbf{0})}=\left(\mathbf{X}^{\mathbf{T}} \mathbf{X}\right)^{-\mathbf{1}}\left(\mathbf{X}^{\mathbf{T}} \mathbf{Y}_{\mathbf{j}}\right)$ dengan $j=0,1,2$. 
2. Membentuk vektor gradien $\mathbf{g}$

$$
\mathbf{g}^{T}\left(\boldsymbol{\theta}_{(m)}\right)_{3(k+1) 1}=\left(\left(\frac{\partial Q}{\partial \boldsymbol{\beta}_{0}}\right)^{T},\left(\frac{\partial Q}{\partial \boldsymbol{\beta}_{1}}\right)^{T},\left(\frac{\partial Q}{\partial \boldsymbol{\beta}_{2}}\right)^{T}\right)_{\theta=\theta(m)}^{T}
$$

3. Membentuk matriks Hessian $\mathbf{H}$

$$
\mathbf{H}\left(\boldsymbol{\theta}_{(m)}\right)_{3(k+1) x 3(k+1)}=\left[\begin{array}{ccc}
\frac{\partial^{2} Q}{\partial \boldsymbol{\beta}_{0} \partial \boldsymbol{\beta}_{0}^{T}} & \frac{\partial^{2} Q}{\partial \boldsymbol{\beta}_{0} \partial \boldsymbol{\beta}_{1}} & \frac{\partial^{2} Q}{\partial \boldsymbol{\beta}_{0} \partial \boldsymbol{\beta}_{2}} \\
& \frac{\partial^{2} Q}{\partial \boldsymbol{\beta}_{1} \partial \boldsymbol{\beta}_{1}^{T}} & \frac{\partial^{2} Q}{\partial \boldsymbol{\beta}_{1} \partial \boldsymbol{\beta}_{2}} \\
\text { simetris } & & \frac{\partial^{2} Q}{\partial \boldsymbol{\beta}_{2} \partial \boldsymbol{\beta}_{2}^{T}}
\end{array}\right]_{\boldsymbol{\theta}=\boldsymbol{\theta}(m)}
$$

4. Memasukkan nilai ke dalam $\hat{\boldsymbol{\theta}}_{(\mathbf{0})}$ elemen-elemen vektor $\mathbf{g}$ dan matriks $\mathbf{H}$, sehingga diperoleh vektor $\mathbf{g}\left(\hat{\boldsymbol{\theta}}_{(0)}\right)$ dan matriks $\mathbf{H}\left(\hat{\boldsymbol{\theta}}_{(0)}\right)$.

5. Mulai dari $m=0$ dilakukan iterasi pada persamaan

$\hat{\boldsymbol{\theta}}_{\mathbf{j}_{(m+1)}}=\hat{\boldsymbol{\theta}}_{\mathbf{j}(m)}-\mathbf{H}^{-1}\left(\hat{\boldsymbol{\theta}}_{(m)}\right) \mathbf{g}\left(\hat{\boldsymbol{\theta}}_{(m)}\right)$

Nilai $\hat{\boldsymbol{\theta}}_{(m)}$ merupakan sekumpulan penaksir parameter yang konvergen saat iterasi ke-m.

6. Jika belum mendapatkan penaksiran parameter yang konvergen, maka dilanjutkan kembali ke langkah 5 hingga iterasi ke $m=m+1$. Iterasi akan berhenti apabila nilai dari $\left\|\hat{\boldsymbol{\theta}}_{(m+1)}-\hat{\boldsymbol{\theta}}_{m}\right\| \leq \varepsilon, \varepsilon$ adalah bilangan yang sangat kecil.

\subsection{Pengujian Parameter Regresi Poisson Bivariat untuk Kovarian Merupakan Fungsi dari Variabel Bebas}

Untuk menentukan nilai statistik uji, terlebih dahulu ditentukan dua buah fungsi likelihood yang berhubungan dengan model regresi yang diperoleh. Fungsi-fungsi likelihood yang dimaksud adalah $L(\hat{\Omega})$ yaitu nilai maximum likelihood untuk model yang lebih lengkap dengan melibatkan variabel prediktor dan $L(\hat{\omega})$, yaitu nilai maximum likelihood untuk model sederhana tanpa melibatkan variabel prediktor. Salah satu metode yang dapat digunakan untuk menentukan statistik uji dalam pengujian parameter menggunakan metode Maximum Likelihood Ratio Test (MLRT) dinotasikan dengan :

$$
\Lambda=\frac{L(\hat{\omega})}{L(\hat{\Omega})}
$$

Hipotesis yang digunakan adalah :

$$
H_{0}: \beta_{j 1}=\beta_{j 2}=\ldots=\beta_{j 8}=0
$$

$H_{1}$ : paling sedikit ada satu $\beta_{j l} \neq 0 ; j=0,1,2 ; l=1,2, \ldots, 8$

Himpunan parameter di bawah $\mathrm{H}_{0}$ adalah $\omega=\left\{\beta_{00}, \beta_{10}, \beta_{20}\right\}$, dengan fungsi likelihood sebagai berikut :

$$
\begin{aligned}
& L(\omega)=\prod_{i=1}^{n} f\left(y_{i} ; \beta_{00} ; \beta_{10} ; \beta_{20}\right) \\
& L(\omega)=\prod_{i=1}^{n}\left(\exp \left(e^{\beta_{00}}-e^{\beta_{10}}-e^{\beta_{20}}\right) . W_{i}\right)
\end{aligned}
$$


Nilai $W_{i}=\sum_{k=0}^{\min \left(y_{1 i}, y_{2 i}\right)} \frac{\left(e^{\beta_{10}}-e^{\beta_{00}}\right)^{y_{1 i}-k}\left(e^{\beta_{20}}-e^{\beta_{00}}\right)^{y_{2 i}-k}\left(e^{\beta_{00}}\right)^{k}}{\left(y_{1 i}-k\right) !\left(y_{2 i}-k\right) ! k !}$

$L(\hat{\omega})=\max _{\omega} L(\omega)$

$L(\hat{\omega})=\prod_{i=1}^{n}\left(\exp \left(e^{\hat{\beta}_{00}}-e^{\hat{\beta}_{10}}-e^{\hat{\beta}_{20}}\right) \cdot W_{i}\right)$

Nilai $W_{i}=\sum_{k=0}^{\min \left(y_{1 i}, y_{2 i}\right)} \frac{\left(e^{\hat{\beta}_{10}}-e^{\hat{\beta}_{00}}\right)^{y_{1 i}-k}\left(e^{\hat{\beta}_{20}}-e^{\hat{\beta}_{00}}\right)^{y_{2 i}-k}\left(e^{\hat{\beta}_{00}}\right)^{k}}{\left(y_{1 i}-k\right) !\left(y_{2 i}-k\right) ! k !}$

Himpunan parameter di bawah populasi adalah $\Omega=\left\{\beta_{j 0}, \beta_{j 1}, \ldots, \beta_{25} ; j=0,1,2\right\}$, sehingga fungsi likelihoodnya sebagai berikut :

$$
\begin{aligned}
& L(\Omega)=\prod_{i=1}^{n} f\left(y_{i} ; \boldsymbol{\beta}_{0} ; \boldsymbol{\beta}_{1} ; \boldsymbol{\beta}_{2}\right) \\
& L(\Omega)=\prod_{i=1}^{n}\left(\exp \left(e^{\mathbf{x}_{\mathbf{i}}{ }^{T} \boldsymbol{\beta}_{0}}-e^{\mathbf{x}_{\mathbf{i}}{ }^{T} \boldsymbol{\beta}_{1}}-e^{\mathbf{x}_{\mathbf{i}}^{T} \boldsymbol{\beta}_{2}}\right) W_{i}\right)
\end{aligned}
$$

dengan

$$
\begin{aligned}
& W_{i}=\sum_{k=0}^{\min \left(y_{1 i}, y_{2 i}\right)} \frac{\left(e^{\mathbf{x}_{\mathbf{i}}^{T} \boldsymbol{\beta}_{\mathbf{1}}}-e^{\mathbf{x}_{\mathbf{i}}^{T} \mathbf{\beta}_{\mathbf{0}}}\right)^{y_{1 i}-k}\left(e^{\mathbf{x}_{\mathbf{i}}^{T} \boldsymbol{\beta}_{2}}-e^{\mathbf{x}_{\mathbf{i}}^{T} \mathbf{\beta}_{\mathbf{0}}}\right)^{y_{2 i}-k}}{\left(y_{1 i}-k\right) !\left(y_{2 i}-k\right) !} \cdot \frac{\left(e^{\mathbf{x}_{\mathbf{i}}^{T} \mathbf{\beta}_{\mathbf{0}}}\right)^{k}}{k !} \\
& L(\hat{\Omega})=\max _{\Omega} L(\Omega) \\
& L(\hat{\Omega})=\prod_{i=1}^{n}\left(\exp \left(e^{\mathbf{x}_{\mathbf{i}}^{T} \hat{\boldsymbol{\beta}}_{\mathbf{0}}}-e^{\mathbf{x}_{\mathbf{i}}^{T} \hat{\boldsymbol{\beta}}_{\mathbf{1}}}-e^{\mathbf{x}_{\mathbf{i}}^{T} \hat{\boldsymbol{\beta}}_{2}}\right) \cdot W_{i}\right)
\end{aligned}
$$

dengan

$$
W_{i}=\sum_{k=0}^{\min \left(y_{1 i}, y_{2 i}\right)} \frac{\left(e^{\mathbf{x}_{\mathbf{i}}^{T} \hat{\mathbf{\beta}}_{\mathbf{1}}}-e^{\mathbf{x}^{T} \hat{\mathbf{\beta}}_{\mathbf{0}}}\right)^{y_{1 i}-k}\left(e^{\mathbf{x}^{T} \hat{\boldsymbol{\beta}}_{2}}-e^{\mathbf{x}_{\mathbf{i}}^{T} \hat{\boldsymbol{\beta}}_{\mathbf{0}}}\right)^{y_{2 i}-k}}{\left(y_{1 i}-k\right) !\left(y_{2 i}-k\right) !} \cdot \frac{\left(e^{\mathbf{x}_{\mathbf{i}}^{T} \hat{\mathbf{\beta}}_{\mathbf{0}}}\right)^{k}}{k !}
$$

sehingga diperoleh

$$
\begin{aligned}
& D(\hat{\boldsymbol{\beta}})=-2 L n\left[\frac{L(\hat{\omega})}{L(\hat{\Omega})}\right] \\
&=2[\ln L(\hat{\Omega})-\ln L(\hat{\omega})] \\
& D(\hat{\boldsymbol{\beta}})=2\left[\begin{array}{l}
\left(\sum_{i=1}^{n} \exp \left(\mathbf{x}_{\mathbf{i}}{ }^{T} \boldsymbol{\beta}_{\mathbf{0}}\right)-\sum_{i=1}^{n} \exp \left(\mathbf{x}_{\mathbf{i}}{ }^{T} \boldsymbol{\beta}_{1}\right)-\sum_{i=1}^{n} \exp \left(\mathbf{x}_{\mathbf{i}}{ }^{T} \boldsymbol{\beta}_{2}\right)+\sum_{i=1}^{n} \ln W_{i}\right)- \\
\left(\sum_{i=1}^{n} \exp \left(\beta_{00}\right)-\sum_{i=1}^{n} \exp \left(\beta_{10}\right)-\sum_{i=1}^{n} \exp \left(\beta_{20}\right)+\sum_{i=1}^{n} \ln W_{i}\right)
\end{array}\right]
\end{aligned}
$$

$D(\hat{\boldsymbol{\beta}})$ adalah devians model regresi poisson bivariat dengan menggunakan pendekatan distribusi chi-square dengan derajat bebas $v$ dan $\mathrm{H}_{0}$ ditolak jika $D(\hat{\boldsymbol{\beta}})>\chi^{2}{ }_{(\alpha ; v)}$, dengan $v$ adalah derajat bebas yang diperoleh dari banyaknya parameter model di bawah populasi dikurangi banyaknya parameter di bawah $\mathrm{H}_{0}$. 


\subsection{Pemodelan Regresi Poison Bivariat Kematian Bayi Dan Kematian Ibu di Propinsi Jawa Tengah Tahun 2015}

Hipotesis yang digunakan untuk uji serentak pada regresi poisson bivariat adalah sebagai berikut :

$$
H_{0}: \beta_{j 1}=\beta_{j 2}=\ldots=\beta_{j 8}=0
$$

$H_{1}$ : paling sedikit ada satu $\beta_{j l} \neq 0 ; j=0,1,2 ; l=1,2, \ldots, 8$

diperoleh nilai $D(\hat{\boldsymbol{\beta}})$ sebesar 2.385,65 dengan tingkat signifikansi sebesar $5 \%$ yang menghasilkan $\chi_{(0,05 ; 24)}^{2}=36,415$ karena nilai $D(\hat{\boldsymbol{\beta}})$ lebih besar dari $\chi_{(0,05 ; 2)}^{2}$ maka tolak $\mathrm{H}_{0}$ yang artinya minimal ada satu variabel yang berpengaruh terhadap respon. Selanjutnya akan dilakukan pengujian parameter secara parsial. Dalam regresi poisson bivariat akan terbentuk tiga model dengan model $\lambda_{1}$ adalah model untuk jumlah kematian bayi, model $\lambda_{2}$ adalah model untuk jumlah kematian ibu, dan model $\lambda_{0}$ adalah model persamaan dari variabel prediktornya. Estimasi parameter model regresi poisson bivariat adalah sebagai berikut :

Tabel 1 Estimasi Parameter Model Regresi Poisson Bivariat pada Kematian Bayi dan Kematian Ibu di Provinsi Jawa Tengah Tahun 2015

\begin{tabular}{c|rrr|rrr}
\hline \multirow{2}{*}{ Parameter } & \multicolumn{3}{|c|}{ Kematian bayi $\left(\lambda_{1}\right)$} & \multicolumn{3}{c}{ Kematian ibu $\left(\lambda_{2}\right)$} \\
\cline { 2 - 7 } & Estimasi & \multicolumn{1}{c}{ SE } & Z Hitung & Estimasi & SE & Z Hitung \\
\hline$\beta_{0}$ & 10,777 & 4,309 & $2,50^{*}$ & 16,128 & 16,278 & 0,99 \\
$\beta_{1}$ & $-0,074$ & $-0,014$ & $5,35^{*}$ & $-1,908$ & $-0,228$ & $8,38^{*}$ \\
$\beta_{2}$ & 0,008 & 0,034 & 0,24 & 1,257 & $-0,049$ & $-25,48^{*}$ \\
$\beta_{3}$ & $-0,019$ & $-0,005$ & $3,51^{*}$ & $-0,170$ & 0,033 & $-5,16^{*}$ \\
$\beta_{4}$ & 0,041 & 0,001 & $69,01^{*}$ & 0,599 & 0,113 & $5,29^{*}$ \\
$\beta_{5}$ & $-0,001$ & $-0,001$ & $3,86^{*}$ & 0,108 & 0,017 & $6,48^{*}$ \\
$\beta_{6}$ & 0,002 & 0,003 & 0,77 & 0,028 & $-0,019$ & $-1,49$ \\
$\beta_{7}$ & 0,007 & 0,003 & $2,27^{*}$ & $-0,071$ & $-0,001$ & $81,55^{*}$ \\
$\beta_{8}$ & $-0,016$ & $-0,007$ & $2,39^{*}$ & $-0,044$ & $-0,008$ & $5,81^{*}$ \\
\hline
\end{tabular}

*) Signifikan dengan taraf signifikansi $5 \%$

Berdasarkan nilai dari Tabel 4.4 ditunjukkan bahwa model $\lambda_{1}$ (kematian bayi) memiliki nilai $Z$ hitung yang lebih besar daripada $Z$ tabel $(\alpha / 2= \pm 1,96)$ ada enam variabel bebas yang berpengaruh signifikan yaitu, persalinan oleh tenaga kesehatan $\left(\mathrm{X}_{1}\right)$, ibu hamil melaksanakan program $\mathrm{K} 4\left(\mathrm{X}_{3}\right)$, ibu hamil yang mendapatkan tablet Fe3 $\left(X_{4}\right)$, penanganan komplikasi kebidanan $\left(X_{5}\right)$, bayi yang diberi ASI eksklusif $\left(X_{7}\right)$, dan rumah tangga berperilaku hidup bersih dan sehat $\left(\mathrm{X}_{8}\right)$. Sedangkan untuk model $\lambda_{2}$ (kematian ibu) hanya variabel penanganan komplikasi neonatal $\left(\mathrm{X}_{6}\right)$ yang tidak memiliki pengaruh yang signifikan terhadap variabel respon. 
Tabel 2 Estimasi Parameter Model Regresi Poisson Bivariat pada $\lambda_{0}$ Persamaan

\begin{tabular}{c|rrr}
\hline \multirow{2}{*}{ Parameter } & \multicolumn{3}{|c}{$\lambda$ persamaan } \\
\cline { 2 - 4 } & \multicolumn{1}{|c}{ Estimasi } & \multicolumn{1}{c}{ SE } & Z Hitung \\
\hline$\beta_{0}$ & 8,023 & $-13,381$ & $-0,60$ \\
$\beta_{1}$ & $-0,007$ & 0,266 & $-0,03$ \\
$\beta_{2}$ & $-0,015$ & 0,045 & $-0,33$ \\
$\beta_{3}$ & $-0,031$ & $-0,177$ & 0,18 \\
$\beta_{4}$ & 0,009 & 0,039 & 0,22 \\
$\beta_{5}$ & $-0,008$ & $-0,021$ & 0,39 \\
$\beta_{6}$ & 0,004 & 0,007 & 0,62 \\
$\beta_{7}$ & 0,004 & $-0,005$ & $-0,84$ \\
$\beta_{8}$ & $-0,009$ & $-0,003$ & $3,43^{*}$ \\
\hline
\end{tabular}

Pada model $\lambda_{0}$ hanya variabel rumah tangga berperilaku hidup bersih dan sehat $\left(\mathrm{X}_{8}\right)$ yang berpengaruh signifikan terhadap variabel respon. Sehingga dari hasil semua estimasi parameter di peroleh model sebagai berikut :

$\hat{\lambda}_{1}{ }^{*}=\exp \left(10,777-0,074 X_{1}+0,008 X_{2}-0,019 X_{3}+0,041 X_{4}-0,001 X_{5}+0,002 X_{6}+\right.$ $\left.0,007 X_{7}-0,016 X_{8}\right)$

$\hat{\lambda}_{2}{ }^{*}=\exp \left(16,128-1,908 X_{1}+1,257 X_{2}-0,170 X_{3}+0,599 X_{4}+0,108 X_{5}+0,028 X_{6}-\right.$ $\left.0,071 X_{7}-0,044 X_{8}\right)$

$\hat{\lambda}_{0}{ }^{*}=\exp \left(8,023-0,007 X_{1}-0,015 X_{2}-0,031 X_{3}+0,009 X_{4}-0,008 X_{5}+0,004 X_{6}+\right.$ $\left.0,004 X_{7}-0,009 X_{8}\right)$

Pada kasus kematian bayi, setiap penambahan $1 \%$ jumlah persalinan oleh tenaga kesehatan $\left(X_{1}\right)$ maka akan melipatgandakan rata-rata jumlah kematian bayi sebesar 0,93 kali dari rata-rata jumlah kematian bayi semula jika variabel lain tidak dilibatkan dalam model. Setiap penambahan $1 \%$ jumlah ibu hamil melaksanakan program K4 $\left(X_{3}\right)$ maka akan melipatgandakan rata-rata jumlah kematian bayi sebesar 0,98 kali dari rata-rata jumlah kematian bayi semula jika variabel lain tidak dilibatkan dalam model. Setiap penambahan $1 \%$ jumlah rumah tangga berperilaku hidup bersih dan sehat $\left(X_{8}\right)$ maka akan melipatgandakan rata-rata jumlah kasus HIV sebesar 0,98 kali dari rata-rata jumlah kematian bayi semula jika variabel lain tidak dilibatkan dalam model.

Pada kasus kematian ibu, setiap penambahan $1 \%$ jumlah persalinan oleh tenaga kesehatan $\left(X_{1}\right)$ maka akan melipatgandakan rata-rata jumlah kematian ibu sebesar 0,15 kali dari rata-rata jumlah kematian ibu semula jika variabel lain tidak dilibatkan dalam model. Setiap penambahan $1 \%$ jumlah ibu hamil melaksanakan program K4 $\left(X_{3}\right)$ maka akan melipatgandakan rata-rata jumlah kematian ibu sebesar 0,84 kali dari rata-rata jumlah kematian ibu semula jika variabel lain tidak dilibatkan dalam model. 


\section{Simpulan dan Saran}

Berdasarkan hasil yang telah diperoleh, estimasi parameter model regresi poisson bivariat regression menggunakan metode maximum likelihood (MLE). Hasil yang diperoleh dari estimasi parameter tersebut tidak close form sehingga perlu dilakukan dengan metode iterasi Newton-Raphson. Pada pengujian hipotesisnya menggunakan metode maximum likelihood rasio test (MLRT) dengan membandingkan nilai antara likelihood di bawah $\mathrm{HO}$ dan likelihood dibawah populasi. Model Regresi Poisson Bivariat pada kematian bayi dan kematian ibu di Provinsi Jawa Tengah Tahun 2015, model $\lambda_{1}$ (kematian bayi) ada enam variabel bebas yang berpengaruh signifikan yaitu, persalinan oleh tenaga kesehatan $\left(X_{1}\right)$, ibu hamil melaksanakan program $\mathrm{K} 4\left(\mathrm{X}_{3}\right)$, ibu hamil yang mendapatkan tablet $\mathrm{Fe} 3\left(\mathrm{X}_{4}\right)$, penanganan komplikasi kebidanan $\left(X_{5}\right)$, bayi yang diberi $A S I$ eksklusif $\left(X_{7}\right)$, dan rumah tangga berperilaku hidup bersih dan sehat $\left(X_{8}\right)$. Sedangkan untuk model $\lambda_{2}$ (kematian ibu) hanya variabel penanganan komplikasi neonatal $\left(\mathrm{X}_{6}\right)$ yang tidak memiliki pengaruh yang signifikan terhadap variabel respon.

\section{Daftar Pustaka}

Cameron, A.C. dan Trivedi, P.K. (1998), Regression Analysis of Count Data, Cambridge University Press, USA.

Chou, N. dan Steenhard, D. (2011), "Bivariate Count Data Regression Models - A SAS® Macro Program”, Proceedings SAS Global Forum 2011, paper 355-2011.

[Dinkes] Dinas Kesehatan Propinsi Jawa Tengah. (2015). Profil Kesehatan Propinsi Jawa Tengah. Semarang : Dinkes Jateng.

Gurmu, S. dan Elder, J. (2007) "A simple bivariate count data regression model." Economics Bulletin, Vol.3, No. 11, hal. 1-10.

Karlis, D. dan Ntzoufras, I. (2005). Bivariate Poisson and Diagonal Inflated Bivariate Poisson Regression Models in R. Journal of Statistical Software, Vol 14, 1-36.

Long, J. S. (1997), Regression Models for Categorical and Limited Dependent Variables. Number 7 in Advance Quantitive Techniques in The Social Sciences, Sage Publications, California. 